\title{
A Complete Formal Semantics of x86-64 User-Level Instruction Set Architecture
}

\author{
Sandeep Dasgupta \\ University of Illinois at \\ Urbana-Champaign \\ USA \\ sdasgup3@illinois.edu
}

\author{
Daejun Park \\ Runtime Verification, Inc. \\ USA \\ dpark69@illinois.edu
}

\author{
Theodoros Kasampalis \\ University of Illinois at \\ Urbana-Champaign \\ USA \\ kasampa2@illinois.edu
}

\author{
Vikram S. Adve \\ University of Illinois at \\ Urbana-Champaign \\ USA \\ vadve@illinois.edu
}

\author{
Grigore Roşu* \\ University of Illinois at \\ Urbana-Champaign \\ USA \\ grosu@illinois.edu
}

\begin{abstract}
We present the most complete and thoroughly tested formal semantics of x86-64 to date. Our semantics faithfully formalizes all the non-deprecated, sequential user-level instructions of the x86-64 Haswell instruction set architecture. This totals 3155 instruction variants, corresponding to 774 mnemonics. The semantics is fully executable and has been tested against more than 7,000 instruction-level test cases and the GCC torture test suite. This extensive testing paid off, revealing bugs in both the x86-64 reference manual and other existing semantics. We also illustrate potential applications of our semantics in different formal analyses, and discuss how it can be useful for processor verification.
\end{abstract}

CCS Concepts - Theory of computation $\rightarrow$ Program reasoning; • Hardware $\rightarrow$ Simulation and emulation; • Software and its engineering $\rightarrow$ Formal language definitions.

Keywords x86-64, ISA specification, Formal Semantics

ACM Reference Format:

Sandeep Dasgupta, Daejun Park, Theodoros Kasampalis, Vikram S. Adve, and Grigore Roşu. 2019. A Complete Formal Semantics of x86-64 User-Level Instruction Set Architecture. In Proceedings of the 40th ACM SIGPLAN Conference on Programming Language Design and Implementation (PLDI '19), June 22-26, 2019, Phoenix,

\footnotetext{
${ }^{*}$ Also with Runtime Verification, Inc., USA.

Permission to make digital or hard copies of all or part of this work for personal or classroom use is granted without fee provided that copies are not made or distributed for profit or commercial advantage and that copies bear this notice and the full citation on the first page. Copyrights for components of this work owned by others than ACM must be honored. Abstracting with credit is permitted. To copy otherwise, or republish, to post on servers or to redistribute to lists, requires prior specific permission and/or a fee. Request permissions from permissions@acm.org.

PLDI '19, fune 22-26, 2019, Phoenix, AZ, USA

(c) 2019 Association for Computing Machinery.

ACM ISBN 978-1-4503-6712-7/19/06 ..\$15.00

https://doi.org/10.1145/3314221.3314601
}

AZ, USA. ACM, New York, NY, USA, 16 pages. https://doi.org/10. $1145 / 3314221.3314601$

\section{Introduction}

The $\mathrm{x} 86-64$ instruction set architecture (ISA) is one of the most complex and widely used ISAs on servers and desktops, and ensuring the correctness of the x86-64 binary code is important. The ability to directly reason about the binary code is desirable, not only because it allows to analyze the binary even when the source code is not available (e.g., legacy code or malware), but also because it avoids the need to trust the correctness of compilers [22, 63].

A formal semantics of $\mathrm{x} 86-64$ is required for formal reasoning about binary code, one of the strongest ways to ensure its correctness. An executable semantics is especially powerful because it allows direct testing to gain confidence in the definitions of the semantics, and also because it can allow additional tools based on symbolic execution, like deductive verification and symbolic test generation. Completely formalizing the semantics of $x 86-64$, however, is challenging especially due to the complexity and the sheer number of instructions that are informally specified in approximately 3,000-page standard [11].

Existing Semantics for $x 86-64$ To date, to the best of our knowledge, despite several explicit attempts [32, 33, 37] and other related systems $[13,35,36,41,42]$, no complete formal semantics of x86-64 exists that can be used for formal reasoning about x86 binary programs. Heule et al. [37] presented a formal semantics of x86-64, but it covers only a fragment ( $47 \%)$ of all instructions; as the authors of [37] candidly admitted, their synthesis methodology proved insufficient to add the remaining instructions primarily due to limitations of the underlying synthesis engine. Moreover, their semantics misses certain essential details (Section 3.6 \& 4). Goel et al. [32, 33], on the other hand, specified a formal semantics in the ACL2 proof assistant [38], allowing to reason about functional correctness, but their semantics covers 
only a small fragment ( $\sim 33 \%)$ of all user-level instructions. There also have been several attempts $[20,24,35,60]$ to indirectly describe the x86-64 semantics, where they define an intermediate language (IL), specify the IL semantics, and translate x86-64 to the IL. This indirect semantics, however, may not be general enough to be used for different types of formal analyses, since the IL might be designed with specific purposes in mind, not to mention that the translation may miss certain important details of the instruction behaviors. Refer to Section 7 for a more detailed comparison to existing semantics.

Our Approach We present the most complete and thoroughly tested formal semantics of user-level x86-64 assembly instructions ${ }^{1}$ to date. We employed the $\mathbb{K}$ framework [59] (Section 2.1) as our formalism medium to leverage its capability of deriving various correct-by-construction formal analysis tools directly from the language semantics. We took Heule et al. [37]'s semantics (Section 2.2) as our starting point to avoid duplicating the formalization effort. We made several corrections or improvements to this semantics, to improve both soundness and efficiency. We automatically translated their semantics into $\mathbb{K}$, and cross-checked the translated semantics against the original using an SMT solver. We manually specified the semantics of the remaining instructions faithfully consulting the Intel manual [11] to obtain the complete semantics. A manual specification may sound like a daunting effort at first, but the fact that (1) x86-64 is largely stable and changes slowly over time, and (2) the state-of-the-art synthesis techniques for language semantics (notably, Strata [37] and Hasabnis et al. [35, 36]) suffer from scalability and/or faithfulness issues (see Section 3.2 and 7 for details), makes the effort worth undertaking.

Like closely related previous work $[33,37]$, we omit the relaxed memory model of x86-64 and thus the concurrencyrelated operations. Modelling concurrency is a complex but relatively orthogonal problem in the presence of small-step operational semantics, as shown in prior work [48, 57], where they have integrated their memory model with a small subset of 32-bit x86 instruction set. We believe that integrating such a memory model into our instruction semantics is a promising direction toward rigorously reasoning about realworld programs running on modern multiprocessors. We leave it for future work.

Contributions Below are our primary contributions.

Completeness. We present the most complete formal semantics of x86-64 to date. Specifically, our semantics formalizes all the user-level instructions of the x86-64 Haswell ISA (that is, 3155 instructions covering 774 mnemonics [11]),

\footnotetext{
${ }^{1}$ The current work do not include a formal model of the binary instruction decoder. Note that, all future references of x86-64 "program(s)" or "instructions(s)", in the context of our model, are meant to refer to the "assembly language programs(s)" or "assembly instruction(s)".
}

except deprecated ones (336 instructions), the AES cryptography extensions (35 instructions), and the system \& concurrency-specific instructions (210 instructions) (Section 3.1).

Faithfulness. Being executable, the semantics of each instruction has been thoroughly tested against 7,000+ test cases using the co-simulation method (Section 4). We found errors in both the x86-64 standard document and other existing semantics including the baseline semantics (Section 4).

Usability \& portability. We illustrate the potential of our semantics to be used for formal analyses such as deductive program verification and program equivalence checking (Section 5). The $\mathbb{K}$ framework also enables one to represent our semantics as SMT theories, which allows others to easily reuse our semantics for their own purposes. Indeed, we have translated our semantics to Stoke [27] which can serve as a drop-in replacement of Heule et al.'s semantics [37] and can immediately benefit tools built on Stoke (e.g., [54]).

Our formal semantics is publicly available at [26].

\subsection{Challenges in Formalizing x86-64}

Size and Complexity The x86-64 ISA has a large number of instructions, partly because of a large number of complex instructions and partly because it keeps most of the legacy and deprecated instructions $(\sim 336+)$ for the sake of backwards compatibility. It consists of 996 mnemonics, and each mnemonic admits several variants, depending on the types (i.e., register, memory, or constant) and the size (i.e., the bit-width) of operands.

Inconsistent Instruction Variants Some variants have divergent behaviors more than the difference of their type and size. For example, movsd, one of the 128-bit SSE instructions, has very different behaviors depending on whether the type of the source operand is register or memory; it clears the higher 64 bits of the target register only when the source type is memory. Indeed, we revealed bugs in other semantics due to their incorrect generalization of the variants' behavior (Details in Section 3.6, Instruction Variants).

Ambiguous Documentation The x86-64 reference manual informally explains the instruction behaviors, leaving certain details unspecified or ambiguous, which required us to consult with an actual processor implementation to clarify such details. Completely formalizing the vast number of instructions with carefully identifying all the corner cases from the informal document, thus, is highly non-trivial.

Undefined Behaviors The x86-64 standard also admits undefined behaviors that are implementation-dependent. Many instructions ( $32^{2}$ out of 996 mnemonics) have undefined behaviors: their output values of the destination register or the

\footnotetext{
${ }^{2}$ These numbers are obtained by parsing the official manual "Volume 2: Instruction Set Reference" and cross checked with projects [16, 58] investing similar efforts.
} 
\%rflags register are undefined in certain cases. That is, the processor is free to choose any behavior in undefined cases.

Many existing semantics, however, simply "define" the undefined behaviors by following a specific behavior taken by a processor implementation. This approach is problematic because they do not capture all possible behaviors of different processor implementations. Indeed, we found discrepancies between existing semantics in specifying the undefined behaviors, where different semantics are valid only for different groups of processors. That is, such semantics are not adequate to formally reason about universal properties (e.g., portability) of a program that need to be satisfied for all standard-conforming processors. For example, the parity flag \%pf is undefined in the logical-and-not instruction andn, where the processor implementation is allowed to either update the flag value (to 0 or 1 ), or keep the previous value. We found, e.g., that Remill [13] updates the flag with 0 , whereas Radare [20] keeps it unmodified. Identifying and faithfully specifying all of the undefined behaviors, thus, are desirable but challenging.

In our semantics, we faithfully modeled the undefined value as a unique symbol (called undef) whose value is nondeterministically decided each time within the proper range. These nondeterministic values are enough to capture and formally reason about all possible behaviors of the instructions for different processors (and even any future, standardconforming processor).

\section{Preliminaries}

Here we provide background on the $\mathbb{K}$ framework and the Strata project [37] (used for our baseline semantics).

\subsection{K Framework}

$\mathbb{K}[59]$ is a framework for defining formal language semantics. Given a syntax and a semantics of a language, $\mathbb{K}$ generates a parser, an interpreter, as well as formal analysis tools such as model checkers and deductive program verifiers, at no additional cost. Using the interpreter, one can test their semantics immediately, which significantly increases the efficiency of semantics developments. Furthermore, the formal analysis tools facilitate formal reasoning about the given language semantics. This helps in terms of both applicability of the semantics and engineering the semantics itself.

We refer the reader to $[55,59]$ for details. In a nutshell, in $\mathbb{K}$, a language syntax is given using conventional Backus-Naur Form (BNF). A language semantics is given as a parametric transition system, specifically a set of reduction rules over configurations. A configuration is an algebraic representation of the program code and state. Intuitively, it is a tuple whose elements (called cells) are labeled and possibly nested. Each cell represents a semantic component, such as the memory or the registers. A special cell, named $\mathrm{k}$, contains a list of computations to be executed. A computation is essentially a program fragment, while the original program is flattened into a sequence of computations. A rule describes a onestep transition between configurations, giving semantics to language constructs. Rules are modular; they mention only relevant cells that are needed in each rule, making many rules far more concise and easy to read than in some other formalisms.

\subsection{Strata Project}

Strata [37] automatically synthesized formal semantics of 1905 instruction variants (representing 466 unique mnemonics) of the x86-64 Haswell ISA. The algorithm to learn the formal semantics of an instruction, say IS, starts with a small set of instructions, called base set $B$, whose semantics are known and trusted; a set of test inputs $T$, and the output behavior of IS obtained by executing IS on T. Then Stoke [58] is used to synthesize instruction sequences which contain instructions from B and match the behavior of IS for all test cases in T. Given two such generated instruction sequences IS and IS', their equivalence is decided using an SMT solver and the trusted and known semantics from the base set. If the two sequences are semantically distinct, then the model produced by the SMT solver is used to obtain an input that distinguishes IS and IS', and t is added to T. This process of synthesizing instruction sequence candidates and accepting or rejecting them based on equivalence checking with previous candidates, is repeated until a threshold is reached, which in their implementation is based on the number of accepted instruction sequences.

For each instruction, Strata manifested its semantics in terms of two related artifacts. The first artifact is an instruction sequence and the second is a set of SMT formulas in the bit-vector theory, one for each output register. The second is obtained by symbolically executing the first.

\section{Formalization of x86-64 Semantics}

This section presents how we get the complete semantics of all the user-level instructions. Section 3.1 details the scope of our work. Section 3.6 mentions how we leverage the information available in Strata, our baseline semantics. Section 3.3 explains how we formalize our model in $\mathbb{K}$.

\subsection{Scope of the Work}

We support all but a few non-deprecated user-level instructions. The support includes 3155 total variants of the Haswell x86-64 ISA (representing 774 out of 996 unique mnemonics). The entire implementation took 8 man-months, with the lead author having prior experience in binary decompilation and strong familiarity with the x86-64 architecture and documentation. Below is a summary of the instruction categories that we support. 
General-Purpose. These implement data-movement, arithmetic, logic, control-flow, string operations (including fastand repeated- string operations).

Streaming SIMD Extensions (SSE) \& subsequent extensions (SSE-2, SSE-3, SSE-4.1, SSE-4.2). Instructions in this category operate on integer, string or floating-point values stored in 128-bit xmm registers. Among other things, the category features instructions related to conversions between integer and floating-point values with selectable rounding mode, and string processing.

Advanced Vector Extensions $(A V X) \&$ subsequent extensions (Fused-Multiply-Add (FMA) \& AVX2). These instructions operate on integer or floating-point values stored in 256-bit ymm registers; a majority of which are promoted from SSE instruction sets. Additionally, the category features enhanced functionalities specific to AVX \& AVX2, like broadcast/permute, vector shift, and non-contiguous data fetch operations on data elements.

16-bit Floating-Point Conversion (or F16C). These instructions implement conversions between single-precision (32bit) and half-precision (16-bit) floating-point values.

Instructions which are not included in the current scope of work are: (1) System-level instructions, which are related to the operating system, protection levels, I/O, cache lines, and other supervisor instructions; (2) x87 \& MMX instructions, consisting of legacy floating-point and vector operations, respectively, which are now superseded by SSE; (3) Concurrency-related operations, including atomic operations and fences; and (4) Cryptography instructions, which support cryptographic processing specified by Advanced Encryption Standard (AES). We note that while there is no inherent limitation in supporting the above instructions with our approach, the system-level instructions require to formulate an abstraction of different architectures and operating systems, which is a significant effort that is orthogonal to the presented effort of formalizing the user-level instructions.

\subsection{Overview of the Approach}

Briefly, our approach is as follows. We first defined the machine configuration and underlying infrastructure in the $\mathbb{K}$ framework, in order to define, execute and test the x86-64 semantics. To leverage previous work as much as possible, we took the semantic rules of all the instructions supported in Strata, which amounts to about $60 \%$ of the instructions in scope, in the form of SMT formulas. We corrected, improved or simplified many of the baseline rules. We then translated these SMT formulas from Strata into $\mathbb{K}$ rules using a script, and tested the resulting rules by comparing with the Strata rules using $Z 3$. These steps give us a validated initial set of semantic rules in $\mathbb{K}$ for about $60 \%$ of the target instructions (our "baseline" set).

We attempted to extend the stratification approach in Strata to define additional rules automatically, in two ways: (i) augmenting their base set B, and (ii) constraining the

$$
\left\langle\langle\mathrm{K}\rangle_{\mathrm{k}}\left\langle\mathrm{ID}_{\text {regname }} \mapsto \text { Value }\right\rangle_{\text {regstate }}\langle\text { Address } \mapsto \text { Value }\rangle_{\text {memstate }} \cdots\right\rangle_{\mathrm{T}}
$$

Figure 1. Program Configuration

search space manually using knowledge of instruction behaviors. Both these attempts failed; they worked only for a few instructions, but in general, we found them to be impractical. Specifically, we added 58 base instructions to the base set, and learned the semantics of 70 new instructions, which are variants of the added instructions, in 20 minutes, but no more even after we kept running for two days. Also, we tried constraining the search space by manually populating it with relevant instructions. The lesson we learned from these experiments is, getting the right set of base instructions or a constrained search space for a complex instruction need an insight about the semantics of that instruction itself. We found that the effort to extract such information from the manual is about the same as manually defining that instruction.

We then manually added $\mathbb{K}$ rules for the remaining $40 \%$ of the target instructions by systematically translating their description of the Intel manual into $\mathbb{K}$ rules, in some cases cross-referencing against semantics available in Stoke. The outcome was a complete formal specification of user-level x86-64 in $\mathbb{K}$.

We validated this semantics in three ways, as described in Section 4. First, we use the $\mathbb{K}$ interpreter to execute the semantics of each instruction for 7,000+ test inputs (each input is a processor state configuration) and compared the output directly with the hardware behavior for the same instruction. Second, we repeated this experiment using the applicable programs in the GCC C-torture tests [7]. Third, we compared against the semantics defined in the Stoke project for about 330 instructions that were omitted in Strata (thus not included in our baseline), using an SMT solver.

These validation experiments uncovered bugs in the Intel manual, in Strata's simplification rules, and in the Stoke semantics. All these bugs were reported to the authors, and most have been acknowledged and some have been fixed. The details are in Section 4.

\subsection{Program Configuration}

Defining a language semantics in $\mathbb{K}$ requires defining the program configuration, the semantics of how programs are evaluated (i.e., the execution environment), and the semantics of the statements or instructions. We begin with the configuration.

The $\mathbb{K}$ configuration of a running x86-64 program is shown in Figure 1. The cells are represented using angle brackets. The outer $T$ cell contains the cells used during program 
evaluation. The inner $\mathrm{k}$ cell contains a list of computations to be executed. Below we describe the two other inner cells. ${ }^{3}$

Register State. The regstate cell contains a map from registers or flag names to values. Note that, all the values or addresses, stored in registers, memory (described next) or flags, are represented as bit-vectors which are depicted in this paper as W'V, and interpreted as a bit-vector of size W and value $V$. The register names include the sixteen general purpose registers, \%rip, and the sixteen SIMD registers. The value mapped to a register name is a 64-width bit-vector (or a 256-width one for the SIMD registers). Values for sub-register variants are derived from the register values by extracting the relevant bits. We store individual flag names (mapped to a bit-vector value of width 1) as opposed to a 64-bit rflags register. Every access (read/write) of \%rflags retrieves the entries in the regstate map for the individual flags.

Memory State. Our memory model is inspired by previous efforts $[29,42]$. The memstate cell is a map from 64-bit addresses to bytes, which specifies the byte-addressable memory ${ }^{4}$, but our implementation is flexible enough to use alternative memory representations with addressing of 2-byte or 4-byte quantities. Our memory layout is "flat", in which all available memory locations can be addressed, but we do have logical partitions ${ }^{5}$ of the memory into sections like code, data and stack. The following is an example snapshot of a memory state, holding a 4-bytes integer value 65535 :

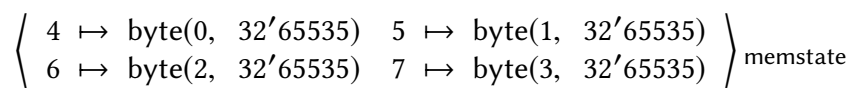

Here the memory address 4 stores the $0^{\text {th }}$ byte of the bitvector $32^{\prime} 65535$, the address 5 stores the $1^{\text {st }}$ byte, and so on. When memory is read, requested bytes are aggregated according to the size of the memory access.

\subsection{Semantics of Execution Environment}

We now give the reader a flavor of our semantics, by discussing a few of the roughly 5,200 rules $^{6}$ that we defined to model the entire semantics. We first explain the semantics of the execution environment, which involves all the machinery used for executing x86-64 programs. We will explain the semantics of individual instructions in the next section.

The execution of an x86-64 program begins with initialization of the configuration with the following contents of the $\mathrm{k}$ cell.

$$
<\mathrm{k}>\text { \$PGM: Instructions } \curvearrowright \text { fetch }</ \mathrm{k}>
$$

The symbol $\curvearrowright$ is used to separate the computations in the $\mathrm{k}$ cell and ": $T$ " to represent the type of a term.

\footnotetext{
${ }^{3}$ We omit other auxiliary cells (marked by “... ”) for the simplicity of the presentation.

${ }^{4}$ Byte-addressability allows the model to specify both aligned and unaligned accesses in the same principle.

${ }^{5}$ These abstractions are useful for executing x86-64 programs.

${ }^{6} \mathrm{Each}$ rule is $17 \mathrm{LOC}$ on average, and the total size is $15 \mathrm{~KB}$ of text.
}

Concisely, the semantics of execution of an x86-64 program involves initializing the memory by reading the program instructions ( $\$ P G M)$, from the $k$ cell, one at a time until all the instructions are loaded in memory. The memoryloaded instructions are then fetched one at a time, using the fetch computation, to get executed. The instruction to be executed next is pointed to by the instruction pointer register \%rip.

Next, we describe the rule applied to initialize memory with instructions one at a time.

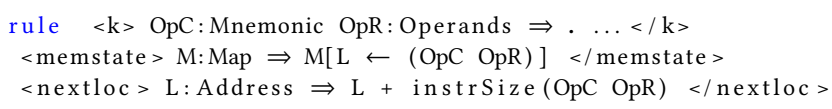

The $\mathrm{k}$ cell contains the instruction to be processed next. Mnemonic and Operands denote the types of the terms used to represent an instruction. The ' $\Rightarrow$ ' symbol represents a reduction (i.e., a transition relation). A cell without the ' $\Rightarrow$ ' symbol means that it is read but not changed by the rule. $\mathbb{K}$ allows us to use "." to represent an empty computation and "..." to match the portions of a cell that are neither read nor written by the rule. The above rule essentially stores each instruction in memory, which is modeled as a map, at an address $L$ given by the nextloc ${ }^{7}$ cell. Subsequently, the nextloc cell gets updated to an appropriate address used for storing the next instruction. Once the entire program is loaded, the fetch-and-execute cycle starts, which is realized by the following rule:

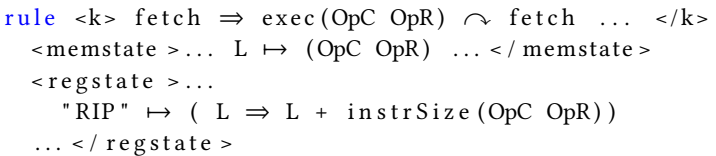

The rule above says that if the next thing to be evaluated is a fetch computation (referred in the rule as fetch), then one should match \%rip in the environment to find its value $L$ in regstate, where $L$ is matched in memstate to find the mapped instruction. The mapped instruction is then put at the head of the $\mathrm{k}$ cell to be computed next, using a rule exec for execution (defined later), along with the fetch computation to be executed in order. The rule also updates the value of \%rip to point to the following instruction. The execution will be terminated when there is no instruction stored in the memory at the address pointed to by \% rip. $^{8}$

\subsection{Semantics of Individual Instructions}

Here we explain how we define the semantics of an instruction in $\mathbb{K}$ using a running example of logical-and-not andnq $-4(\% r s p), \% r b x, \%$ rax, which performs a bitwise

\footnotetext{
${ }^{7}$ The nextloc cell is a auxiliary cell that holds the next memory location to store an instruction, which we omit in the program configuration (Figure 1) for the sake of simplicity.

${ }^{8}$ While initializing the stack section of memory, we store an invalid address just before the entry-point function as return address. When the entry point function returned, the invalid return address is popped out of the stack and stored in \%rip leading to program termination.
} 
logical AND of inverted source register operand (\%rbx) with the source memory operand (-4(\%rsp)) and writes the result to destination register \%rax. Additionally the instruction affects all the 6 status flags (\%sf, \%zf, \%of, \%cf, \%af and \%pf).

The semantics of most of the instructions can be modeled broadly in 3 phases: (1) read the data from source operand(s), which could be a register, memory or constant value; (2) operate on the data based on the mnemonic; and (3) write the result(s) to destination operand(s), which could be a register or memory. An instruction may exercise some or all of the above phases.

Read from Source Operand(s) Instruction in the running example reads from register (\%rbx) and memory (-4(\%rsp)) operands. A read from register is modeled as a lookup with register name in the regstate map and subsequent read of the mapped value or, for a sub-register, a portion of it. The semantics of register read can be defined as:

rule <k> getRegisterVal $(\mathrm{R}: \mathrm{R} 64) \Rightarrow \mathrm{BV}_{\mathrm{r}} \ldots</ \mathrm{k}>$

$<$ regstate $>\ldots \mathrm{R} \mapsto \mathrm{BV}_{\mathrm{r}} \ldots</$ regstate $>$

In the context of the running example, this rule is applied when the current computation (at top of the $\mathrm{k}$ cell) is a 64bit register lookup, appeared as getRegisterVal(\%rbx), and regstate contains a register with name "RBX". This rule resolves the register lookup to the mapped bit-vector value $\mathrm{BV}_{\mathrm{r}}$ (or $\mathrm{B} \mathrm{V}_{\mathrm{RBX}}$ for the running example).

A read from memory involves computing the effective address in the memory, looking-up that address in memory, and reading requested bytes from memory if the memory access is within allowed range. The following rule is applied to compute the effective address:

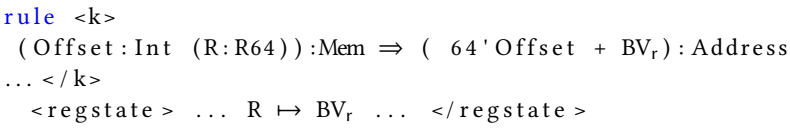

The term to the left of $\Rightarrow$ shows the memory addressing expression, of type Mem, at the top of k cell, which gets reduced to an effective memory address (or EA). The EA for the memory operand used in the running example is $\left(64^{\prime}-4\right.$ $\left.+\mathrm{BV}_{\mathrm{rsp}}\right)$ and is used to do memory read access. The rule for memory read access is responsible to read a memory value of requested number of bits (64-bits for the current example) starting from the EA.

Operate on Data The rules for operating on operands will be different for each instruction based on the mnemonic. For example, the mnemonic andnq requires logical-and-not operation to be computed on the operands.

Write to Destination Operand(s) The example instruction writes the result to a destination register \%rax. Also, the flags sf and zf are updated based on the result; of and $\mathrm{cf}$ are cleared, and af and pf are undefined. The rule shown in Figure 2 realizes the destination write operation, where memVal ${ }_{64}$ and $\mathrm{BV}_{\mathrm{r}}$ represents the 64-bit data values evaluated using the respective rules for reading register and

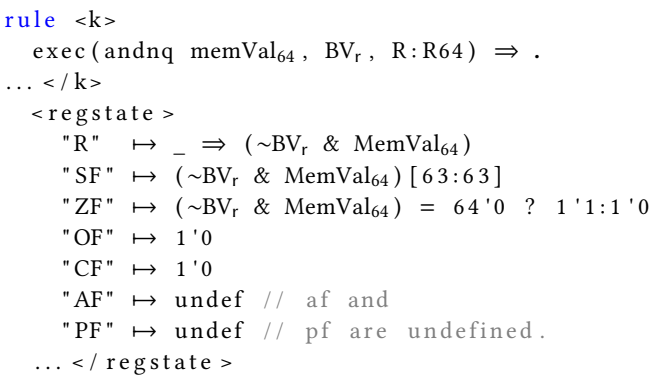

Figure 2. Example instruction semantics of andn

memory operands (mentioned above). The operator "[i:j]" extracts bits i down to $\mathrm{j}$ from a bit-vector of size $\mathrm{n}$, yielding another bit-vector of size $\mathrm{i}-\mathrm{j}+1$, assuming that $\mathrm{n}>\mathrm{i} \geq \mathrm{j}$ $\geq 0$. The operator " $\&$ " implements bit-wise "and" operation. The rule associated with memory write is similar to that for memory read and is skipped here.

A x86-64 program is modeled as a list of instructions and its semantics is given by composing the semantics of its constituents.

\subsection{Constructing the x86-64 Semantics}

Systematic Translation of Strata Rules to K As mentioned in the introduction, we leverage the Strata [37] semantics to develop our complete semantics, to minimize the overall effort. We systematically translated their semantics into $\mathbb{K}$. Specifically, Strata offers the semantics of 1905 instruction variants as SMT formulas specifying the behavior of output registers. For each instruction, we converted the SMT formulas that Strata provides to a $\mathbb{K}$ specification using a simple script ( $\sim 500$ LOC).

To validate the translation, we generated SMT formulas from the translated $\mathbb{K}$ specifications (using APIs provided by the $\mathbb{K}$ framework), and use the Z3 SMT solver to check their equivalence to the corresponding formulas provided by Strata. While translating and validating their semantics, we found various issues that we had to fix to establish our baseline semantics. Below we describe the issues we found in Strata.

Status Flags We found that Strata omitted to specify the $\%$ af flag behaviors, as the flag is not commonly used. However, we faithfully specified the semantics of all the status flags in the \%rflags register, even if some of them are not commonly used, since they may affect the overall program's behavior in some tricky cases, and we do not want to miss any of such details when formally reasoning about the x86-64 programs.

Instruction Variants Strata essentially provides the semantics of the register instructions, assuming that the semantics of the memory and immediate instruction variants can 
be obtained by generalizing the register instructions ${ }^{9}$. However, we found that certain memory instructions cannot be inferred by simply generalizing their corresponding register instructions. For example, for movsd, one of the 128-bit SSE instructions, its register variant has quite different semantics from the memory variant. Below are their pseudo-code semantics:

$$
\begin{aligned}
& \text { Semantics of Register Variant Semantics of Memory Variant } \\
& \text { (movsd \%xmm1 , \%xmm) (movsd (\%rax), \%xmm0) } \\
& \text { S1. XMM0[63:0] XMM1[63:0] S1.XMM0[63:0] } \leftarrow \text { MEM_ADDR[63:0] } \\
& \text { S2. XMM0[127:64] (Unmodified) S2. XMM0[127:64] } \leftarrow 0
\end{aligned}
$$

As seen, only the memory instruction clears the higher 64 bits of the destination register, which cannot be inferred from the register instruction behavior that does not touch the higher bits at all. We found that another 128-bit SSE instruction, movss, has the same generalization issue. For the other instructions, we obtained the memory and immediate variants by generalizing the register variants, and validated the generalization by co-simulating the inferred semantics against a processor.

Immediate Instruction Variants There are 118 immediate instruction variants (over the 8-bit constants) that do not have corresponding register instructions. For those immediate instructions, Strata provides the instruction semantics for each individual constant, resulting in 30,208 $(=118 \times 256)$ formulae $^{10}$ for the immediate instructions' semantics. We generalized the set of formulae for each immediate instruction into a single semantic rule. We validated our generalization by cross-checking the generalized semantics with the original using the SMT solver.

Formula Simplification Due to the nature of the stratification, Strata provides complex formulae for certain instructions. We simplified those complex formulae by either applying some simplification rules or manually translating into simpler ones. Then we validated the simplification by checking the equivalence between them using the SMT solver. For example, the original Strata-provided formula for shrxl \%edx, \%ecx, \%ebx consists of 8971 terms (including the operator symbols), but we could simplify it to a formula consisting of only 7 terms.

\section{Validation of Semantics}

A formal semantics is of limited use if one cannot generate confidence in its correctness. In this section, we describe how we establish that confidence in our model.

\footnotetext{
${ }^{9}$ Generalization is based on a hypothesis that the memory or immediate variants will behave identically, on their operands, with corresponding register variant.

${ }^{10}$ Indeed, Strata explicitly provides only 19,783 formulae by randomly sampling $\sim 168$ constants out of 256 , in average, for each immediate instruction, assuming that the remaining 10,425 formulae can be inferred.
}

\subsection{Co-Simulations against Hardware}

Empowered by the fact that we can directly execute the semantics using the $\mathbb{K}$ framework, we validated our model by co-simulating it against a real machine. During co-simulation, we execute a machine program on the processor as well as on our $\mathbb{K}$ model and compare the execution results after every instruction. In this work, we co-simulated our model against two Intel implementations that were available to the authors at the time of writing: "Intel Xeon CPU E3-1505M v6" and "Intel Xeon CPU E5-2640 v4". We admit that testing the model against other hardwares (such as AMD) would contribute to more thorough validation of our model, having the potential of revealing flaws in those implementations and/or additional imperfections in the manual as well, which we leave as future work.

We first describe our test-infrastructure and then talk about individual validation experiments and results.

Test Harness During co-simulations, we need to make sure that the program must be instrumented similarly both on our model and the real hardware. We use the GNU Debugger [10] to instrument programs on hardware. We developed instrumentation tools based on $\mathbb{K}$ framework to gain similar capabilities for our model. Using these tools we can record the output state (including memory) after the execution of each instruction. To facilitate debugging, in the event when the output states do not match, we developed a tool which points to the first instant when the output states diverge and this saves debugging time.

The co-simulation experiments are done in the following two phases: (1) Instruction level validation: testing individual instructions, and (2) Program level validation: testing a combination of instructions as a part of real-world programs.

Instruction Level Validation The goal here is to execute individual instructions both on hardware and our model using test inputs and then compare the output states.

$\mathbb{K}$ already has matured library support for bit-vector, integer and floating-point theories. We use bit-vectors to implement the values stored in registers or memory. Depending upon an instruction mnemonic, these values can be interpreted as integers (signed/unsigned) or floating-point values (with various precisions). We augmented the library support in $\mathbb{K}$ framework to interpret these bit-vectors accordingly. With that support, we can execute and hence test instructions implementing various floating-point operations including conversions (to and from integer/floating-point values) with selectable rounding modes (e.g. Nearest, +Inf, -Inf and Truncate).

Test Inputs A test input is a CPU state which includes values for all registers, flags and memory. Our test input set contains more than 7, 000 inputs, obtained from the following sources: (1) In section 2.2, we mentioned that Strata starts its algorithm with a set of test inputs which keeps on augmenting 
itself during the process of stratification. We used the final augmented test-suite of 6630 test inputs, (2) While testing instructions implementing floating-point operations, we found that many of the test inputs are representing a NaN or Infinity and it makes no sense to test with such instances. We did our best effort by manually generating more than 100 unique floating-point values by consulting the IEEE floating-point arithmetic standard [1], (3) We used the $(\sim 100)$ test-inputs offered by Remill [13], and (4) We manually implemented a regression test-suite worth of around 200 test-inputs which we accumulated over the course of the project.

Note that, each instruction semantics consists of one or more semantic rules, where those rules cover different cases of the instruction behaviors (including the undefined ones). We ensure that our test inputs are sufficient enough to trigger all of the semantic rules, achieving the full "semantic-rule" coverage.

Results Our current implementation of the fused-multiplyadd operation ${ }^{11}$ incorrectly rounds the operation twice (after multiplication and addition) as opposed to once. As a result, we encountered floating-point precision issues while testing instructions implementing those operations (vfmadd132pd). This is a limitation of the underlying $\mathbb{K}$ library and more details about this limitation can be found in Section 6 .

While performing the validation tests, we encountered various cases where the output state obtained by executing the semantics on our model does not agree with that of the hardware execution. The instruction semantics in our model is either based on the Strata project (for the part we borrowed) or on the Intel manual. A difference in the output state could mean a bug in Strata's instruction semantics or in our interpretation of the Intel manual or in the Intel manual itself. We found many bugs in our interpretation which we fixed, but in other cases, we found issues in Intel manual and Strata project.

Inconsistencies Found in the Intel Manual Here are inconsistencies found during development and testing. According to the manual, the semantics of vpsravd \%xmm3,\%xmm $2, \%$ xmm 1 seems to depend on the lower 100 bits of \%xmm3, whereas the actual hardware execution suggests that it should depend on the lower 128 bits. Similar inconsistencies are found in instructions with mnemonics vpsllvd, vpsllvq, vpsravd. Also, we found misleading typos related to instructions with opcodes vpsravw, vpsravd, vpsravq, packsswb. All these findings were reported and acknowledged by Intel as issues in the manual [4].

Inconsistencies Found in Strata's Simplification Rules While testing the instructions specifications borrowed from Strata, we found inconsistent behaviors with the actual hardware.

\footnotetext{
${ }^{11}$ According to the standard IEEE-754-2008 [1] (Definition 2.1.28), the operation fused-multiply-add $(x, y, z)$ computes $x \times y+z$ as if with unbounded range and precision, rounding only once to the destination format.
}

Moreover, the inconsistencies were discovered in the formulas of floating-point instructions. This is not surprising because Strata models the floating-point instructions as uninterpreted functions which cannot be executed or tested on hardware. Their semantics are executable in our definition though, and thus we were able to test them thoroughly. Note that Strata generates the formulas for these instructions by symbolically executing the corresponding learned instruction sequences followed by a formula simplification pass. Therefore, errors in those formulas can be due to bugs either in the symbolic execution engine or in the simplification stage. Our testing shows that the second is true with the following evidence. The simplification rule add_double $(A, 0)==A$ does not hold for $A=-0.0$. Same for add_single. These were reported [8]. Also, the simplification rule sub_double $(\mathrm{A}, \mathrm{A})==0$ does not hold for $\mathrm{A}=\mathrm{NaN}$. Same is true for sub_single. We found this bug in the branch of Stoke which is used in Strata. But this has been already fixed in the latest Stoke branch.

Program Level Validation The goal here is to test the combination of instructions as part of real-world programs and we chose to use GCC C-torture tests [7] for this purpose. Specifically, we used the tests inside the "testsuite/gcc.ctorture/execute" directory for GCC version 8.1.0. There are originally 1576 tests, which we compiled using the GCC switches "-O0 -march=haswell -S -mlong-double-64 -mno80387”. The last two switches avoid generating x87 instructions that are not in the scope of work. We had to exclude 6 programs containing system-level instruction prefetchnta, which require modeling caches, which we currently do not support. Many test-cases involve C-library functions, like malloc, fprintf, most of whose semantics are modeled in $\mathbb{K}$. As our support of C-library functions is not exhaustive, we have to exclude 22 programs containing un-supported functions like vfprintf and vsprintf, which we plan to support in future. This brings us to a grand total of 1548 viable tests, which are all tested. Out of those, we found that there are 293 cases where floating-point instructions are used covering 35 unique floating-point operations. Moreover, all the testcases together cover about 963 instruction variants, covering $30 \%$ of our supported instructions. As before, we executed each program on the processor as well as on our model and compared the output state after every instruction, which matches in all the cases ${ }^{12}$.

\subsection{Comparing with Stoke}

Stoke $[58]^{13}$ contains manually written semantics for $\sim 1764$ x86-64 instruction variants, a large fraction (81\%) of which

\footnotetext{
${ }^{12}$ Note that none of test-cases include floating-point instructions implementing fused-multiply-addition, which we already acknowledged to have precision issues.

${ }^{13}$ Recall that Stoke is a stochastic super-optimizer leveraged by Strata for stochastic search.
} 
is also supported by Strata. The remaining fraction is exclusive to Stoke. Comparing with Stoke provides an additional crosscheck on our model. Moreover, these manually written formulas are based on a similar model of the CPU state to ours, which makes it easier to compare them against ours by using an SMT solver. While doing so we found inconsistencies between the two formalisms in a total of 16 mnemonics (42 instruction variants), and after careful analysis, identified these as errors in the Stoke specification of instruction semantics, as follows.

Inconsistencies Found in Stoke First, for instructions like addsubpd \%xmm1, \%xmm2, the order of addition and subtraction specified by Stoke is opposite to the one specified in the Intel Manual. Same is true with the mnemonic addsubps. (Found in 12 instruction variants.)

Second, the instruction pslld \%xmm1, \%xmm2 implements a logical left shift of packed data by a count specified in $\% \mathrm{xmm} 1$. Stoke's specification vectorized the operand \% $\mathrm{xmm} 1$ which is incorrect according to the manual. Similar issues were found in instructions implementing the logical right shift operations on packed data. (Found in 18 instructions.)

Third, cvtsi2sdl \%eax, \%xmm1 and vcvtsi2sdl \%eax, $\% x m m 0, \% x m m 1$ are respectively SSE- and AVX-versions of the instruction to convert a double-word (32-bit) integer to a scalar single-precision floating-point value. According to the manual, in the AVX-version, the destination bits 127 - 64 of the register $\% \mathrm{xmm} 1$ are updated to the corresponding bits in the first source operand \%xmm0. This is in contrast to the SSE-version of the instruction where the destination bits $127-64$ should remain unmodified. Stoke specifies the semantics of the AVX-version similar to the SSE-version, which is incorrect. (Found in 4 instruction variants.)

Finally, some instructions, like imulb \%al, which drive flag registers to an undefined state are not modeled correctly in Stoke. (found in 8 instruction variants)

All these errors were reported and confirmed [5, 6].

\section{Applications}

In this section, we illustrate a few applications of our formal semantics, in addition to the reference model mentioned in the previous section. Our goal here is to explain that our semantics can be used for formal reasoning of x86-64 programs for a wide variety of purposes. For this reason, the applications are illustrative only, not meant to serve as a comprehensive evaluation or make any claim of scalability. Moreover, the reported performance of the applications is not optimized, and there is room for improvement, e.g., by providing custom abstractions and lemmas specific to x86-64, similarly to [49]. However, we believe that each application has the potential to be leveraged into a standalone tool, with its own user interface and case studies, but this is not our goal here. In fact, thanks to the language-parametric nature of $\mathbb{K}$, none of these reasoning approaches can be regarded as novel per se, because they are already used in the context of other languages defined in $\mathbb{K}$ and their implementation is language-semantics agnostic. We begin with a discussion of a use case for hardware verification.

\subsection{Validating Processor Hardware}

Verification is considered one of the most (if not the most) important challenges in modern processor design, for several reasons: (i) the enormous state spaces of modern systems; (ii) the lack of formal specifications in the state-of-practice, (iii) generating high-quality test inputs for simulation, (iv) quantifying/analyzing the extent of coverage of simulation, and (v) generating a complete set of properties for checking. For post-silicon validation, an additional challenge is the difficulty of debugging and diagnosing observed erroneous behaviors. For all these reasons, verification is estimated to use $70 \%$ of the resources and time, while design takes only $30 \%$ [31].

A fully executable formal ISA-level specification such as the one developed here can improve the state of practice in verification in two significant ways.

First, it can provide a reliable specification of the functional behavior of hardware with respect to observable states. This increases confidence in the input tests, for both directed and random test generation. High confidence tests can reduce time and increase focus during debugging, triage and diagnosis efforts. This is especially valuable in post-silicon validation, where observability within the chip is very limited, and functional validation is a key goal. This method can also help post-silicon failure diagnosis by identifying buggy input/output pairs and pinpointing specific erroneous output state bits.

Second, since our method can symbolically execute instructions, it can be used to generate input tests that have high coverage. While such analyses have been done at the detailed RTL level [19, 43, 44], we are unaware of similar tools at the x86-64 ISA level ${ }^{14}$. The most significant advantage of such symbolic execution is the ability to detect corner case or hard to detect bugs [30,44]. This is analogous to finding security vulnerabilities due to corner-case software bugs, illustrated in Section 5.3, but applied to the hardware implementation instead of software. We expect that ISA level symbolic analysis will uncover such subtle and complex bugs due to the higher level of abstraction and greater scalability (in terms of execution lengths) than the RTL analyses.

A closely related challenge is checking the accuracy of ISA specifications, including reference manuals. By using such manual specifications to construct a formal specification, we may uncover errors in the manual specifications. This is

\footnotetext{
${ }^{14}$ It is worth mentioning the work by Martignoni et al. [45] about test-case generation for 32-bit x86 ISA by symbolically executing the instruction implementations in bochs [40] binary emulator. However, floating-point instructions are excluded because the underlying symbolic execution engine does not support them.
} 


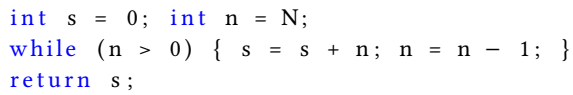

(a) C source code

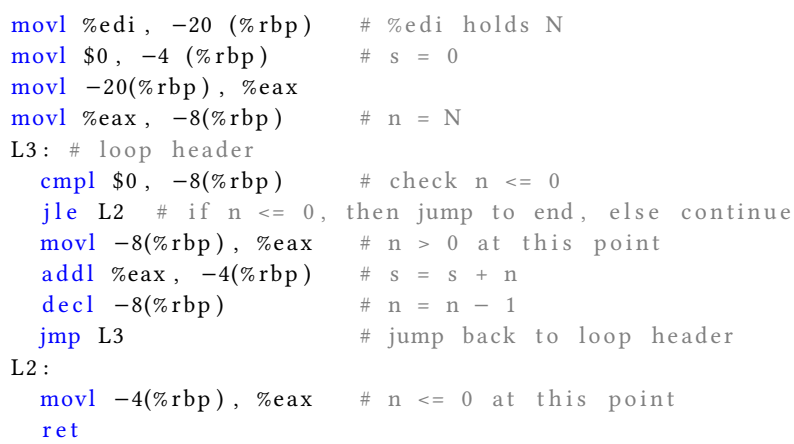

(b) x86-64 assembly code

Figure 3. sum-to-n program

explicitly demonstrated by the two bugs we discovered in the Intel x86-64 manual while performing the instructionlevel validation tests described in Section 4 . These bugs were discovered as a result of running test cases using both the formal semantics generated by reading the manuals and the hardware, and finding a mismatch, then checking the manual specification carefully to determine whether the bug lies in the manuals or in the hardware. However, given an existing semantics, a far more valuable strategy would be to automatically generate human-readable documentation from the formal specification. A basic version of this strategy is likely quite feasible today, and much more sophisticated versions that synthesize illustrative examples and even explanatory text automatically could be possible soon, given recent advances in concolic test generation, program synthesis, and natural language processing.

\subsection{Program Verification}

The $\mathbb{K}$ framework provides a language-parametric, reachability logic theorem prover [53, 62]. We instantiated it with our semantics to generate a correct-by-construction deductive verifier for x86-64 programs. Here, the functional correctness properties are specified as reachability specifications, essentially a pair of pre- and post-conditions for each function. The derived x86-64 verifier uses a sound and relatively complete proof system to prove the given specifications w.r.t. the x86-64 semantics. Like in other deductive verifiers, repetitive constructs such as loops and recursive functions need to be annotated with invariants. The verifier is automatic: it requires only the program, its specification, and the invariants.

To demonstrate that our semantics can be used to verify x86-64 programs, we use the $x 86-64$ verifier to prove the functional correctness of the sum-to-n program as shown in Figure 3. It takes $N$ as input and returns the sum from 1 to $N$. The functional correctness can be essentially described as:

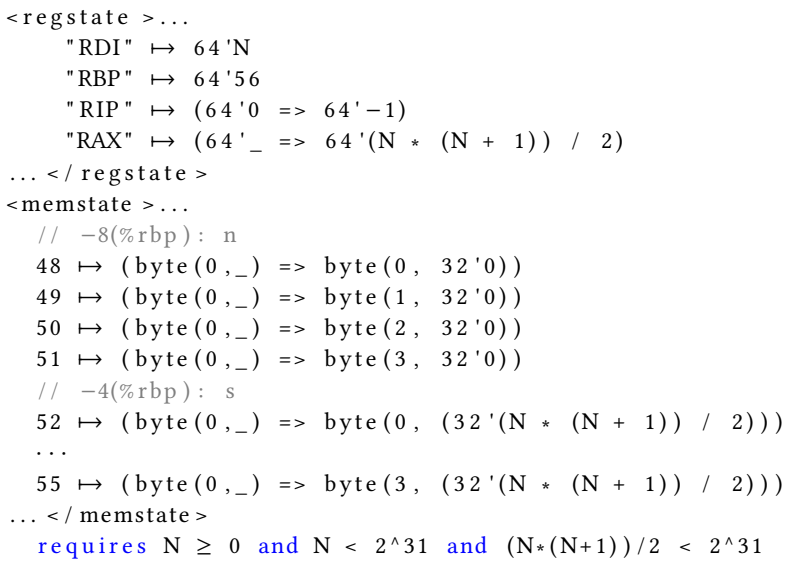

(a) Top-level specification

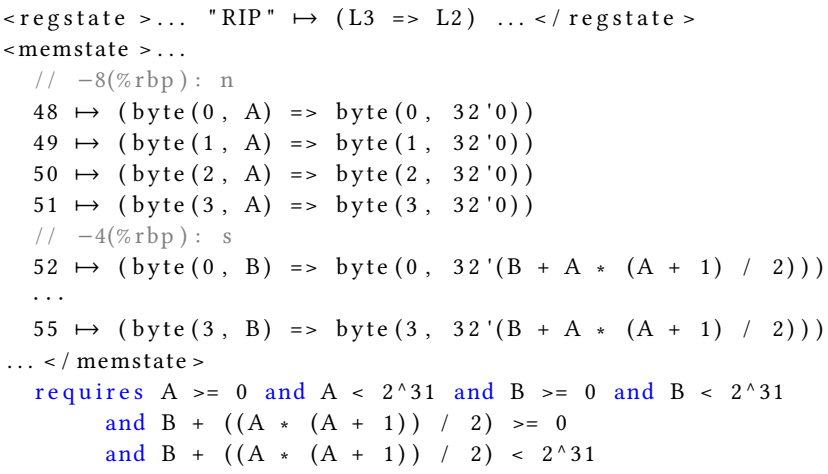

(b) Loop invariant

Figure 4. Specification of sum-to-n program

$\% \operatorname{rax}=\sum_{1}^{N} n=N(N+1) / 2$. We present the actual specification that is fed to the $\mathrm{x} 86-64$ verifier. The specification has two parts: the top-level specification and the loop invariant.

Figure 4(a) shows the functional correctness specification of the sum-to-n program. The regstate cell specifies the relevant registers used in the program, omitting the irrelevant ones denoted by “.... Specifically, it specifies that \%rdi holds the value $N$ without being updated during the program execution, and \%rax will have the expected return value. The memstate cell specifies the relevant part of the memory omitting others (denoted by “...”). It specifies the stack memory addresses $-8(\% \mathrm{rbp})$ and $-4(\% \mathrm{rbp})$ corresponding to $\mathrm{n}$ and $\mathrm{s}$, respectively. The requires clause specifies the condition of $N$ that prevents the arithmetic overflow. Figure 4(b) shows the loop invariant specification. It specifies the behavior of an arbitrary loop iteration. That is, assuming the values of $n$ and s be $A$ and $B$, resp., in the beginning of an arbitrary loop iteration, it specifies their final values in the end of the entire loop execution, which are 0 and $B+A(A+1) / 2$, respectively. Note that when $A=N$ and $B=0$, i.e., the first loop iteration, the loop invariant captures the entire loop behavior. The $\mathbb{K}$ 


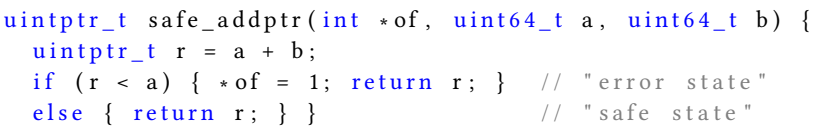

(a) C source code

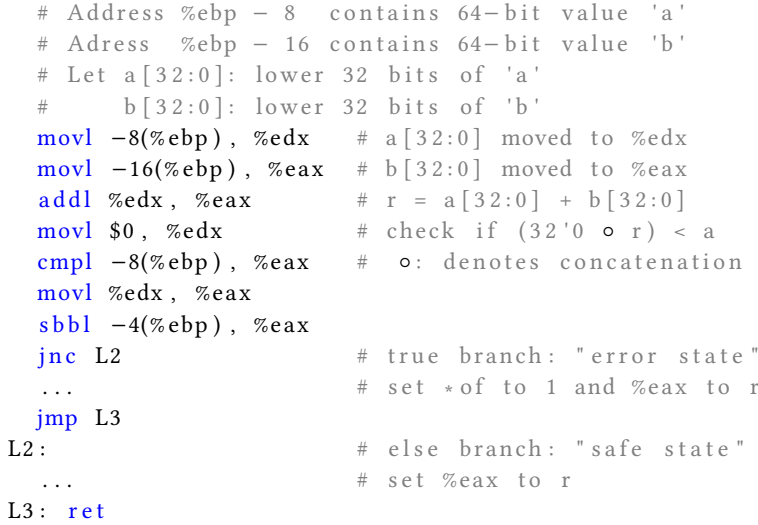

(b) x86-64 assembly code in a 32-bit target

Figure 5. A security vulnerability in the HiStar kernel

verifier takes a minute ${ }^{15}$ to verify the sum-to-n assembly code satisfies the functional correctness specification.

\subsection{Symbolic Execution}

$\mathbb{K}$ automatically derives a correct-by-construction symbolic execution engine from the given semantics. Being instantiated with our semantics, the engine can be used to symbolically execute and explore all possible paths in the given x86-64 program. In this section, we demonstrate how this capability can be used to find a security vulnerability.

Consider the code snippet of the HiStar [64] kernel, as shown in Figure 5(a) ${ }^{16}$, in which the KLEE [25] team found a security vulnerability. The safe_addptr function is supposed to compute the sum of two arguments a and b, setting the flag argument of when the arithmetic overflow occurs during the addition. That is, one of the functional correctness properties is that " $*$ of $=1$ if $a+b>r$ ", where + is the mathematical addition (with no overflow). The functional correctness, however, is not satisfied when the source code is compiled to a 32-bit target, since the size of $r$ becomes 32-bit (uintptr_t) while the sizes of $a$ and $b$ are still 64-bit

\footnotetext{
${ }^{15}$ The application is for illustrative purpose and evaluating its scalability is left to future work. However, we note that the reported verification time would not be a major concern for scalability because of the modularity of deductive verification.

${ }^{16}$ For the simplicity of the presentation, in Figure 5(b), we highlight only the key computations of the assembly compiled from the source. However, in our experiment, the full unmodified compilation is used for the symbolic execution.
}

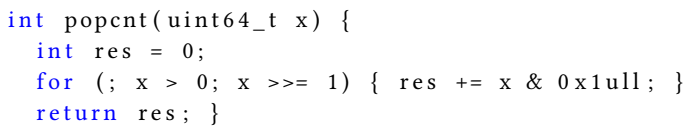

Figure 6. popcnt program

(uint64_t). ${ }^{17}$ A suggested fix [25] is to change the conditional expression from $r<$ a to $r<a \| r<b$.

Using the symbolic execution engine derived from our semantics, we could find (in $\sim 80$ seconds) that, in the assembly code as shown in Figure 5(b), there exists a path that reaches L2 (i.e., the else branch) even if the addition overflow occurs. The (simplified) path condition provided by the symbolic execution engine is:

$$
a+b \geq 2^{32} \wedge\left(a+b \bmod 2^{32}\right) \geq a
$$

where $0 \leq a<2^{64}$ and $0 \leq b<2^{64}$. We asked Z3 to solve the above path condition and it returned a solution (i.e., a concrete input to trigger the security vulnerability): $a=$ $0 x 00000000 \mathrm{ffffffff}$ and $b=0 x f f f f f f f 00000000$.

\subsection{Translation Validation of Optimizations}

$\mathbb{K}$ also provides a program equivalence checker that can be used for the translation validation of compiler optimizations. We derived an x86-64 program equivalence checker from our semantics and used it to validate different optimizations. Figure 6 shows a program that we considered, popcnt, which counts the number of set bits in the given input.

We compiled the program with different optimizations: the GCC compiler optimizations (-O0, -O1, -O2, and -O3), and the Stoke super-optimization. On top of the baseline (-O0), the -O1 optimization produces a code obtained by performing the mem2reg optimization, the -O2 optimization produces one by factoring out the common statement over different branches, ${ }^{18}$ and the -O3 optimization produces the same code with -O2. The Stoke super-optimization translates the assembly code into a single instruction: popcnt \%rdi, \%rax, where \%rdi and \%rax correspond to the input and the return values, respectively.

We validated these optimizations by checking the equivalence between the optimized programs. The equivalence checker symbolically executes each program and compares their return values (i.e., the symbolic expression of the \%rax register value) using $Z 3$. It is able to prove successfully that all optimization variants are equivalent, i.e., to check the correctness of all these optimizations on popcnt.

\footnotetext{
${ }^{17}$ The function call safe_addptr( ${ }^{*}$ of, address, size) is used to validate that an user is allowed to access the memory range specified by the arguments address and size. The access is denied if an overflow occurs. A bug in the overflow detection might be exploited by an attacker to gain an access to a memory region beyond the control of the running process.

${ }^{18}$ Specifically, by performing the common subexpression elimination, followed by certain statement reordering optimization, followed by the strength reduction.
} 
Note that the symbolic execution of the popcnt program does not require an additional annotation about the loop because the number of loop iterations is bound to a constant (i.e., the bit-size of the input, 64$).{ }^{19}$ In general, however, the equivalence checker may require us to provide an additional annotation about loops, which can be automatically generated by augmenting the underlying compiler.

\section{Limitations}

Our limitations mostly include missing features of the x86-64 and execution environment, as follows.

Floating-Point Operations. Our testing shows that we have FP precision issues with instructions implementing the fused-multiply-add operation. This is because the current $\mathbb{K}$ 's floating-point library [12] implementation lacks support of the FMA capabilities of GNU MPFR library [15], which we plan to include in future.

Exceptions. We do not support exceptions, including the FP exceptions. Moreover, we do not distinguish between quiet and signaling NaN, i.e. all NaNs are quiet in our model. When the exception condition is encountered, execution proceeds after setting the exception flag.

Concurrency. Like the closest previous work [33,37], we do not model concurrent semantics or the relaxed memory model as defined by other previous work [48, 57]. Our design, being parameterized on memory model, is amenable to accommodate others', which we plan to achieve in future.

Instruction Decoding. The instruction decoding semantics is not modeled in the current work. However, we want to note that we have formalized the Intel XED disassembler algorithm, and we are working on formalizing the instruction decoding.

\section{Related Work}

There have been many projects that host a formal semantics of x86-64 either as their main contribution or as part of their infrastructure. This section summarizes such previous work and compares it to our formal semantics based on three directions that reflect the primary contributions of our work: completeness, in terms of supported user-level instructions; faithfulness, in terms of whether it is executable and hence can be evaluated with real code execution; and generality, in terms of its applicability to formal reasoning techniques.

Strata [37] uses program synthesis to generate the instruction semantics of X86-64 as SMT bit-vector formulas. Automatically learning the formal semantics of $60 \%$ of the target $\mathrm{x} 86-64$ ISA is impressive, and we leverage this result

\footnotetext{
${ }^{19}$ However, an additional annotation about the loop (i.e., a loop invariant) can be provided to improve the symbolic execution performance. For example, symbolic execution of the popcnt program without the loop invariant requires to iterate the loop 64 times, which takes $\sim 20$ minutes, but it can be reduced to a minute if the loop invariant is provided.
}

in our work. However, the other $40 \%$ of the user-level instructions are not straightforward to automatically learn by their algorithm, mainly due to limitations of the underlying synthesis engine. Moreover, the specifications are executable only for non-floating-point (FP) instructions.

A contemporary work by Roessle et al. [54] presents a method to extract the big step semantics of a binary program using the small step instruction semantics extracted mostly from Strata ${ }^{20}$ plus some manually drafted support for branching instructions and stack operations. Like Strata, their specification is executable only for the non-floatingpoint instructions. Moreover, their work does not aim for completeness of semantics, one of our primary goals.

Goel et al. [33] use the ACL2 theorem prover [38] to model the x86-64 ISA and they support $33 \%$ of all user-level instructions [17], plus some system-level instructions, paging, and segmentation. This list is far from a complete semantic definition of $x 86-64$, but it is still the state-of-the-art in terms of formal analysis applied directly to x86-64 code. It is also an executable definition as demonstrated by its use for simulations. In our work, we do not leverage this definition, since Strata has defined many more instructions.

The CompCert verified compiler [41] includes semantics definitions for all intermediate and target languages used within the compiler, including a definition for 32-bit x86 assembly. The definition is specified in Coq [14] and has been used in a formal setting for proving the correctness of CompCert's compilation step to assembly, as well as outside CompCert, e.g., in proofs relating to the certified concurrent OS kernel CertiKOS [34]. However, this definition focuses on the 32-bit x86 instruction set, which is a subset of the x86-64 instruction set. Moreover, it is part of the trust base for CompCert and it is not clear whether or how it has been tested against an actual processor, whereas Strata and ours have been extensively tested.

TSL [42] is a system that can auto-generate tools for various machine code analyses given a semantics definition of the machine language written in the TSL specification. Such a semantics definition for the integer instructions (i.e., no floating-point instructions) of the 32-bit x86 instruction set is given as part of the project. It is used to generate various tools, including a machine code synthesizer [61]. This definition, to our knowledge, has not been used for formal verification proofs, i.e., to prove whether a given x 86 program meets its specification.

Our semantics, like all the other work cited above, uses a sequential consistency memory model, and not weaker memory models. Existing efforts to specify weaker memory models for x86-64 such as Owens et al. [48] and Sarkar et al. [57], however, suffer from their limited support for instruction semantics (i.e., they consider only a small subset of

\footnotetext{
${ }^{20}$ There are some minor omissions on immediate instructions with 8-bit operands for which Strata learns 256 brute force formulas.
} 
32-bit x86 instruction set). We believe that integrating these two complementary efforts is a promising direction toward rigorously reasoning about real-world programs running on modern multiprocessors (e.g., using the Sail framework as we will describe below).

Sail is another language semantics framework, tailored for describing an instruction-set architecture semantics. Sail has been used to specify the semantics of ARMv8-A, RISC-V, and CHERI-MIPS [21], as well as the semantics of a small subset of $\mathrm{x} 86$ [2]. Sail is similar to the K framework we employed, but $\mathrm{K}$ is far more general-purpose than Sail. Also, the Sail $\mathrm{x} 86$ semantics is much more limited than ours. It describes the semantics of a fragment of 32-bit user-mode x86 instructions, while ours covers also the 64-bit counterpart as well as the floating-point instructions. Sail, however, allows us to integrate a semantic definition with their relaxed memory models $[18,50]$ for concurrency semantics. We believe that (automatically) translating our semantics into Sail ${ }^{21}$ is a promising direction to obtain concurrency semantics and thus enable concurrency reasoning for x86 programs, which we leave as future work.

Overall, the key differentiator of our effort compared to the existing work, as cited above, is that our semantics achieves (A) completeness of supported user-level instructions, (B) faithfulness, and (C) applicability to formal reasoning analyses. In Section 8, we elaborate on our novel approaches that allow us to achieve this unique combination.

There are various binary analysis projects that target x8664 binaries and lift them to a higher-level representation more suitable for the specific analysis. These include Angr [3] using the VEX IR of Valgrind [47], the QEMU [23] emulator using the TCG IR, the software fault isolation tool RockSalt [46] using its own RTL DSL, the disassembler and binary analyzer Radare2 [20] using the ESIL IR [9], the binary analysis tool BAP [24] using the BIL IR, and the static binary translator Remill [56] using LLVM IR [39]. We refer to these semantics as indirect because they give the semantics of the x86-64 binary via the translation to their IR, as opposed to a direct semantics such as ours and the others cited earlier. A direct semantics has significant advantages over an indirect semantics. For example, without the direct semantics of x86-64, we cannot even formulate the correctness of a translator from x86-64 to the IR. Analogously, many programming languages $(\mathrm{C}, \mathrm{C}++$, Java, etc.) have been given direct semantics, instead of indirect semantics by translation to other languages, for formal reasoning at the desired language granularity.

Hasabnis et al. [35, 36] also present an indirect semantics of $x 86-64$, but in contrast to other indirect semantics, they use machine learning [36] and symbolic execution [35] to

\footnotetext{
${ }^{21}$ Indeed, the Sail ARMv8-A semantics is automatically generated from the ARM-internal specification of ARMv8-A [52] written in the ARM's architecture specification language, ASL [51], by using the ASL-to-Sail translator [21].
}

automatically learn the translation of x86-64 instructions to their IR, by extracting knowledge from the hard-coded translation logic of compilers such as GCC. However, as they admitted [35], their semantics omits some important details of x86-64 semantics (e.g., the effect of various instructions on CPU flags), and thus is not sufficient to serve as a solid foundation for rigorous formal analyses of x86-64 binary.

\section{Lessons Learned}

Here we present the lessons we learned during our semantics development, identifying important aspects to be considered, and clarifying best practices for developing a large ISA semantics. We also discuss the novel aspects of our semantics development approach that allow us to obtain a complete and faithful semantics with a practical amount of effort.

Automatic semantics synthesis Most previous efforts in formalizing x86-64 semantics can be categorized based on whether the underlying approach is fully manual $[2,33,41$, 42] or fully automatic [35-37, 54]. We note that none of these approaches, when used in isolation, sufficiently scale to a complete and faithful semantics, as much as ours that combines these complementary approaches so that they benefit from each other.

Section 3.2 reports the challenges we encountered in achieving fully automatic synthesis of the entire x86-64 semantics. Specifically, in a vast instruction set like x86-64, it is common that many instructions can be grouped together where the instructions of each group are similar to each other except for a few differences. An automatic synthesis technique leveraging such a group, such as the stratification approach [37], would effectively synthesize such instruction variants' semantics, provided that the semantics of representative instructions in each group are given in advance. ${ }^{22}$ The problem, however, is that it is non-trivial to properly partition all the instructions into such groups, providing the representative instruction semantics for each group, without a priori knowledge about the semantics of all instructions. The vanilla stratification approach [37] turned out to be not sufficient to solve this dilemma, leaving a substantial part of the semantics unspecified. Thus, we decided to manually provide the information about the partition and representatives, for which we had to consult the manual to obtain knowledge about the remaining part of semantics. Once we obtained the required knowledge, however, we realized that it would be more straightforward to directly turn the knowledge into the semantics than going through the synthesis process, and thus we ended up manually specifying the remaining part of the semantics.

\footnotetext{
${ }^{22}$ For certain complex instructions, the size of their group is very small (i.e., they are quite different to each other), and thus the automatic synthesis would not yield a sufficient gain over the effort of specifying the semantics of their representatives, but we found that the number of such isolated instructions of $\times 86-64$ is small.
} 
Another important step of the semantics synthesis is postprocessing. The generated semantics is often verbose and not necessarily human-readable. The post-processing step is desired to simplify the generated semantics to be succinct, which helps to increase the human-readability as well as to improve the efficiency when being employed in other applications (e.g., the size of SMT formula encoding can be reduced, which can reduce the burden of SMT solvers). For our semantics development, we have written dozens of simplification rules that are fed to the $\mathbb{K}$ framework to simplify the synthesized semantics further (Section 3.6).

Modeling and executing implementation-dependent behaviors The x86-64 ISA standard admits implementationdependent behaviors for certain operations on certain input patterns, that is, each processor implementation can freely choose the execution behavior for each such case (Section 1.1). Faithfully modeling the implementation-dependent behaviors is necessary for the correctness of the semantics. For example, as mentioned in Section 4.2, Stoke [58] does not faithfully model such behaviors, causing certain errors in their semantics that we revealed [5].

There are two natural, faithful ways of specifying implementation dependent behaviors. One is to parameterize the semantics over the implementation-dependent behaviors, and later instantiate it with a profile that describes specific behaviors taken by the processor of interest. This approach is desirable for validating the semantics using concrete execution. Another is to introduce non-determinism in the semantics, which captures a set of different possible behaviors in a single semantics, which is desirable during symbolic interpretation of the ISA code. We note that most of other existing $d i-$ rect $\mathrm{x} 86-64$ semantics employ approaches similar to the ones described above, faithfully modeling the implementationdependent behaviors. For example, Goel et al. [32] models such behaviors using a constraint function which is guaranteed to be unique and non-deterministic, while they employ the aforementioned profile-based approach for concrete execution. TSL [42] makes both approaches available, from which their users can choose.

In our semantics, we faithfully modeled the undefined value as a unique symbol (called undef) whose value is nondeterministically decided each time within the proper range. For validating the semantics, we concretely executed the semantics while the non-deterministic behaviors are represented symbolically using the undef symbol and then we checked if the hardware output is matched by (an instance of) the simulated output.

\section{Employing multiple semantic engineering frameworks} We found that employing multiple semantic frameworks is helpful. Specifically, we employed the two semantic frameworks, $\mathbb{K}$ and Stoke, where we enjoyed all of their (executive) benefits that make it easier for us to write and validate the semantics, and utilize the semantics in various applications.
For example, we wrote the semantics of certain complicated instructions (e.g., pcmpestri, pcmpestrm, and pclmulqdq) in $\mathbb{K}$, as $\mathbb{K}$ provides an easy way to specify behaviors with multiple cases, while Stoke would have required us to write a big nested if-then-else expression, which is not convenient. As another example of the benefits, we used Stoke to validate most of our instruction semantics as Stoke provides an infrastructure ${ }^{23}$ for hardware co-simulation, whereas we employed $\mathbb{K}$ to validate the semantics of floating-point instructions as Stoke does not support executing floating-point operations while $\mathbb{K}$ does.

In order to use the two frameworks interchangeably, we developed a translator between the semantics of the two frameworks. To check the correctness of the translation, we verified equivalence between the original and the translated semantics for each instruction using the Z3 SMT solver.

To summarize, employing multiple frameworks with validated translation between them improved both the ease of specification (using $\mathbb{K}$ ) and ease of validation (using Strata), which expedited our semantics development process and thus significantly contributed to the completeness of our semantics. Moreover, we immediately benefit from all of their formal analysis tools, increasing the applicability of the semantics in various formal reasoning tasks. Existing semantics development efforts (e.g., [33, 37]), however, employ a single framework without utilizing the potential of other frameworks, which otherwise might have improved completeness and/or faithfulness of their semantics with the same amount of effort.

\section{Conclusion}

We have presented the most complete formal semantics of x86-64 user-level instructions to date, and have thoroughly tested it using synthesized test inputs and the GCC torture tests. We have also illustrated several potential uses of the semantics which are realized by the formal analysis tools derived right from the $\mathbb{K}$ specification. The $\mathbb{K}$ framework also enables us to represent a semantics as SMT theories, which other projects can leverage for their own purposes.

\section{Acknowledgments}

We thank the $\mathbb{K}$ team, for their technical support throughout the project, and the Strata developers, for promptly confirming our reported bugs and answering all our questions in great detail. We thank our shepherd, June Andronick, for her diligence and helpful guidance in responding to the reviewers' comments. We are grateful to Alastair Reid and Matthew Fernandez for their invaluable feedback. The work presented in this paper was supported in part by the Office of Naval Research under contract number N00014-17-1-2996, NSF CNS 1619275, and DARPA Sub HRL 17090-181687 US.

\footnotetext{
${ }^{23}$ Indeed, we contributed to their infrastructure as well [27, 28].
} 


\section{References}

[1] 2008. IEEE Std 754-2008 - IEEE Standard for Floating-Point Arithmetic. https://standards.iee.org/findstds/standard/754-2008.html. https: //doi.org/10.1109/IEEESTD.2008.4610935

[2] 2017. Sail x86 ISA model. https://github.com/rems-project/sail/tree/ sail2/x86. Last accessed: May 2, 2019.

[3] 2018. Angr: A powerful and user-friendly binary analysis platform! http://angr.io/. Last accessed: May 2, 2019.

[4] 2018. Bug Reported in Intel Developer Zone: Possible errors in instruction semantics. https://software.intel.com/en-us/forums/ intel-isa-extensions/topic/773342. Last accessed: May 2, 2019.

[5] 2018. Bug Reported in Stoke: Modelling the behavior of flags which may or must take undef values. https://github.com/StanfordPL/stoke/ issues/986. Last accessed: May 2, 2019.

[6] 2018. Bug Reported in Stoke: Semantic bugs. https://github.com/ StanfordPL/stoke/issues/983. Last accessed: May 2, 2019.

[7] 2018. C Language Testsuites: C-torture version 8.1.0. https://gcc.gnu. org/onlinedocs/gccint/C-Tests.html. Last accessed: May 2, 2019.

[8] 2018. Eric Schkufza. Personal communication.

[9] 2018. Evaluable Strings Intermediate Language. https://radare. gitbooks.io/radare2book/content/disassembling/esil.html. Last accessed: May 2, 2019.

[10] 2018. GDB: The GNU Project Debugger. https://www.gnu.org/ software/gdb/. Last accessed: May 2, 2019.

[11] 2018. Intel 64 and IA-32 Architectures Software Developer Manuals. https://software.intel.com/en-us/articles/intel-sdm. Published on October 12, 2016, updated May 18, 2018.

[12] 2018. MPFR Java Bindings. https://github.com/kframework/mpfr-java. Last accessed: May 2, 2019.

[13] 2018. Remill: Library for lifting of $x 86$, amd64, and aarch64 machine code to LLVM bitcode. https://github.com/trailofbits/remill. Last accessed: May 2, 2019.

[14] 2018. The Coq Proof Assistant. https://coq.inria.fr/. Last accessed: May 2, 2019.

[15] 2018. The GNU MPFR Library. https://www.mpfr.org/. Last accessed: May 2, 2019.

[16] 2018. x86 and amd64 Instruction Reference (UnOfficial). http://www. felixcloutier.com/x86/. Last accessed: May 2, 2019.

[17] 2018. X86isa: Implemented-opcodes: Opcodes supported by the x86 model. http://www.cs.utexas.edu/users/moore/acl2/manuals/current/ manual/index-seo.php/X86ISA IMPLEMENTED-OPCODES. Last accessed: May 2, 2019.

[18] 2019. rmem: Executable concurrency models for ARMv8, RISC-V, Power, and x86. https://github.com/rems-project/rmem/. Last accessed: May 2, 2019.

[19] A. Ahmed, F. Farahmandi, and P. Mishra. 2018. Directed test generation using concolic testing on RTL models. In 2018 Design, Automation Test in Europe Conference Exhibition (DATE). 1538-1543. https://doi.org/ 10.23919/DATE.2018.8342260

[20] Sergi Alvarez. 2018. Radare2. https://rada.re/r/. Last accessed: May 2, 2019.

[21] Alasdair Armstrong, Thomas Bauereiss, Brian Campbell, Alastair Reid, Kathryn E. Gray, Robert M. Norton, Prashanth Mundkur, Mark Wassell, Jon French, Christopher Pulte, Shaked Flur, Ian Stark, Neel Krishnaswami, and Peter Sewell. 2019. ISA Semantics for ARMv8-a, RISC-v, and CHERI-MIPS. Proc. ACM Program. Lang. 3, POPL, Article 71 (Jan. 2019), 31 pages. https://doi.org/10.1145/3290384

[22] Gogul Balakrishnan and Thomas Reps. 2010. WYSINWYX: What You See is Not What You eXecute. ACM Trans. Program. Lang. Syst. 32, 6, Article 23 (Aug. 2010), 84 pages. https://doi.org/10.1145/1749608. 1749612

[23] Fabrice Bellard. 2005. QEMU, a Fast and Portable Dynamic Translator. In Proceedings of the Annual Conference on USENIX Annual Technical Conference (ATEC '05). USENIX Association, Berkeley, CA, USA, 41-41. http://dl.acm.org/citation.cfm?id=1247360.1247401

[24] David Brumley, Ivan Jager, Thanassis Avgerinos, and Edward J. Schwartz. 2011. BAP: A Binary Analysis Platform. In Proceedings of the 23rd International Conference on Computer Aided Verification (CAV'11). Springer-Verlag, Berlin, Heidelberg, 463-469. http://dl.acm. org/citation.cfm?id=2032305.2032342

[25] Cristian Cadar, Daniel Dunbar, and Dawson Engler. 2008. KLEE: Unassisted and Automatic Generation of High-coverage Tests for Complex Systems Programs. In Proceedings of the 8th USENIX Conference on Operating Systems Design and Implementation (OSDI'08). USENIX Association, Berkeley, CA, USA, 209-224. http://dl.acm.org/citation. $\mathrm{cfm} ? \mathrm{id}=1855741.1855756$

[26] Sandeep Dasgupta. 2018. Semantics of x86-64 in K. https://github. com/kframework/X86-64-semantics. Last accessed: May 2, 2019.

[27] Sandeep Dasgupta. 2019. Defining semantics of instructions unsupported in Strata/Stoke. https://github.com/StanfordPL/stoke/pull/996. Last accessed: May 2, 2019.

[28] Sandeep Dasgupta. 2019. Improving Stoke ability to debug a circuit. https://github.com/StanfordPL/stoke/pull/997. Last accessed: May 2, 2019.

[29] Chucky Ellison and Grigore Roşu. 2012. An Executable Formal Semantics of C with Applications. In Proceedings of the 39th ACM SIGPLANSIGACT Symposium on Principles of Programming Languages (POPL'12). ACM, 533-544. https://doi.org/10.1145/2103656.2103719

[30] Pedro Fonseca, Kaiyuan Zhang, Xi Wang, and Arvind Krishnamurthy. 2017. An Empirical Study on the Correctness of Formally Verified Distributed Systems. In Proceedings of the Twelfth European Conference on Computer Systems (EuroSys '17). ACM, New York, NY, USA, 328-343. https://doi.org/10.1145/3064176.3064183

[31] Harry D. Foster. 2015. Trends in Functional Verification: A 2014 Industry Study. In Proceedings of the 52nd Annual Design Automation Conference (DAC '15). ACM, New York, NY, USA, 48:1-48:6. https://doi.org/10.1145/2744769.2744921

[32] Shilpi Goel, Warren A. Hunt, and Matt Kaufmann. 2017. Engineering $a$ Formal, Executable x86 ISA Simulator for Software Verification. Springer International Publishing, Cham, 173-209. https://doi.org/10.1007/ 978-3-319-48628-4_8

[33] Shilpi Goel, Warren A. Hunt, Matt Kaufmann, and Soumava Ghosh. 2014. Simulation and Formal Verification of x86 Machine-Code Programs That Make System Calls. In Proceedings of the 14th Conference on Formal Methods in Computer-Aided Design (FMCAD '14). FMCAD Inc, Austin, TX, Article 18, 8 pages. http://dl.acm.org/citation.cfm? id $=2682923.2682944$

[34] Ronghui Gu, Zhong Shao, Hao Chen, Xiongnan Wu, Jieung Kim, Vilhelm Sjöberg, and David Costanzo. 2016. CertiKOS: An Extensible Architecture for Building Certified Concurrent OS Kernels. In Proceedings of the 12th USENIX Conference on Operating Systems Design and Implementation (OSDI'16). USENIX Association, Berkeley, CA, USA, 653-669. http://dl.acm.org/citation.cfm?id=3026877.3026928

[35] Niranjan Hasabnis and R. Sekar. 2016. Extracting Instruction Semantics via Symbolic Execution of Code Generators. In Proceedings of the 2016 24th ACM SIGSOFT International Symposium on Foundations of Software Engineering (FSE 2016). ACM, New York, NY, USA, 301-313. https://doi.org/10.1145/2950290.2950335

[36] Niranjan Hasabnis and R. Sekar. 2016. Lifting Assembly to Intermediate Representation: A Novel Approach Leveraging Compilers. In Proceedings of the Twenty-First International Conference on Architectural Support for Programming Languages and Operating Systems (ASPLOS '16). ACM, New York, NY, USA, 311-324. https: //doi.org/10.1145/2872362.2872380

[37] Stefan Heule, Eric Schkufza, Rahul Sharma, and Alex Aiken. 2016. Stratified Synthesis: Automatically Learning the x86-64 Instruction Set. In Proceedings of the 37th ACM SIGPLAN Conference on Programming Language Design and Implementation (PLDI '16). ACM, New York, NY, USA, 237-250. https://doi.org/10.1145/2908080.2908121 
[38] Matt Kaufmann, J. Strother Moore, and Panagiotis Manolios. 2000. Computer-Aided Reasoning: An Approach. Kluwer Academic Publishers, Norwell, MA, USA.

[39] Chris Lattner and Vikram Adve. 2004. LLVM: A Compilation Framework for Lifelong Program Analysis \& Transformation. In Proceedings of the 2004 International Symposium on Code Generation and Optimization (CGO'04). Palo Alto, California.

[40] Kevin P. Lawton. 1996. Bochs: A Portable PC Emulator for Unix/X. Linux F. 1996, 29es, Article 7 (Sept. 1996). http://dl.acm.org/citation. cfm?id $=326350.326357$

[41] Xavier Leroy. 2009. Formal Verification of a Realistic Compiler. Commun. ACM 52, 7 (July 2009), 107-115. https://doi.org/10.1145/1538788. 1538814

[42] Junghee Lim and Thomas Reps. 2013. TSL: A System for Generating Abstract Interpreters and Its Application to Machine-Code Analysis. ACM Trans. Program. Lang. Syst. 35, 1, Article 4 (April 2013), 59 pages. https://doi.org/10.1145/2450136.2450139

[43] L. Liu and S. Vasudevan. 2011. Efficient validation input generation in RTL by hybridized source code analysis. In 2011 Design, Automation Test in Europe. 1-6. https://doi.org/10.1109/DATE.2011.5763253

[44] Lingyi Liu and Shobha Vasudevan. 2014. Scaling Input Stimulus Generation Through Hybrid Static and Dynamic Analysis of RTL. ACM Trans. Des. Autom. Electron. Syst. 20, 1, Article 4 (Nov. 2014), 33 pages. https://doi.org/10.1145/2676549

[45] Lorenzo Martignoni, Stephen McCamant, Pongsin Poosankam, Dawn Song, and Petros Maniatis. 2012. Path-exploration Lifting: Hi-fi Tests for Lo-fi Emulators. In Proceedings of the Seventeenth International Conference on Architectural Support for Programming Languages and Operating Systems (ASPLOS XVII). ACM, New York, NY, USA, 337-348. https://doi.org/10.1145/2150976.2151012

[46] Greg Morrisett, Gang Tan, Joseph Tassarotti, Jean-Baptiste Tristan, and Edward Gan. 2012. RockSalt: better, faster, stronger SFI for the x86. PLDI: Programming Languages Design and Implementation (2012), 395-404. https://doi.org/10.1145/2254064.2254111

[47] Nicholas Nethercote and Julian Seward. 2003. Valgrind: A Program Supervision Framework. Electronic Notes in Theoretical Computer Science 89, 2 (2003), 44 - 66. https://doi.org/10.1016/S1571-0661(04) 81042-9 RV '2003, Run-time Verification (Satellite Workshop of CAV '03).

[48] Scott Owens, Susmit Sarkar, and Peter Sewell. 2009. A Better x86 Memory Model: X86-TSO. In Proceedings of the 22Nd International Conference on Theorem Proving in Higher Order Logics (TPHOLs '09). Springer-Verlag, Berlin, Heidelberg, 391-407. https://doi.org/10.1007/ 978-3-642-03359-9_27

[49] Daejun Park, Yi Zhang, Manasvi Saxena, Philip Daian, and Grigore Roşu. 2018. A Formal Verification Tool for Ethereum VM Bytecode. In Proceedings of the 2018 26th ACM Joint Meeting on European Software Engineering Conference and Symposium on the Foundations of Software Engineering (ESEC/FSE 2018). ACM, New York, NY, USA, 912-915. https://doi.org/10.1145/3236024.3264591

[50] Christopher Pulte, Shaked Flur, Will Deacon, Jon French, Susmit Sarkar, and Peter Sewell. 2017. Simplifying ARM Concurrency: Multicopyatomic Axiomatic and Operational Models for ARMv8. Proc. ACM Program. Lang. 2, POPL, Article 19 (Dec. 2017), 29 pages. https://doi. org $/ 10.1145 / 3158107$

[51] Alastair Reid. 2016. ARM's Architecture Specification Language. https: //alastairreid.github.io/specification_languages/. Last accessed: May
2, 2019.

[52] Alastair Reid. 2017. Trustworthy specifications of ARM ${ }^{\circledR}$ v8-A and v8-M system level architecture. Proceedings of the 16th Conference on Formal Methods in Computer-Aided Design, FMCAD 2016 (2017), 161-168. https://doi.org/10.1109/FMCAD.2016.7886675

[53] Grigore Roșu and Andrei Ştefănescu. 2012. Checking Reachability using Matching Logic. In Proceedings of the 27th Conference on ObjectOriented Programming, Systems, Languages, and Applications (OOPSLA'12). ACM, 555-574. https://doi.org/citation.cfm?doid=2384616. 2384656

[54] Ian Roessle, Freek Verbeek, and Binoy Ravindran. 2019. Formally Verified Big Step Semantics out of x86-64 Binaries. In Proceedings of the 8th ACM SIGPLAN International Conference on Certified Programs and Proofs (CPP 2019). ACM, New York, NY, USA, 181-195. https: //doi.org/10.1145/3293880.3294102

[55] Grigore Roşu and Traian Florin Şerbănuţă. 2010. An Overview of the K Semantic Framework. Journal of Logic and Algebraic Programming 79, 6 (2010), 397-434. https://doi.org/10.1016/j.jlap.2010.03.012

[56] Andrew Ruef and Artem Dinaburg. 2014. Static Translation of X86 Instruction Semantics to LLVM with McSema. REcon (2014). https: //github.com/trailofbits/mcsema

[57] Susmit Sarkar, Peter Sewell, Francesco Zappa Nardelli, Scott Owens, Tom Ridge, Thomas Braibant, Magnus O. Myreen, and Jade Alglave. 2009. The Semantics of x86-CC Multiprocessor Machine Code. In Proceedings of the 36th Annual ACM SIGPLAN-SIGACT Symposium on Principles of Programming Languages (POPL '09). ACM, New York, NY, USA, 379-391. https://doi.org/10.1145/1480881.1480929

[58] Eric Schkufza, Rahul Sharma, and Alex Aiken. 2013. Stochastic Superoptimization. In Proceedings of the Eighteenth International Conference on Architectural Support for Programming Languages and Operating Systems (ASPLOS '13). ACM, New York, NY, USA, 305-316. https://doi.org/10.1145/2451116.2451150

[59] Traian Florin Şerbănuţa, Andrei Arusoaie, David Lazar, Chucky Ellison, Dorel Lucanu, and Grigore Roşu. [n. d.]. The K Primer (version 3.2). Technical Report.

[60] Yan Shoshitaishvili, Ruoyu Wang, Christopher Salls, Nick Stephens, Mario Polino, Audrey Dutcher, John Grosen, Siji Feng, Christophe Hauser, Christopher Kruegel, and Giovanni Vigna. 2016. SoK: (State of) The Art of War: Offensive Techniques in Binary Analysis. (2016).

[61] Venkatesh Srinivasan and Thomas Reps. 2015. Synthesis of machine code from semantics. Proceedings of the 36th ACM SIGPLAN Conference on Programming Language Design and Implementation - PLDI 2015 (2015), 596-607. https://doi.org/10.1145/2737924.2737960

[62] Andrei Stefănescu, Daejun Park, Shijiao Yuwen, Yilong Li, and Grigore Roşu. 2016. Semantics-based Program Verifiers for All Languages. In Proceedings of the 2016 ACM SIGPLAN International Conference on Object-Oriented Programming, Systems, Languages, and Applications (OOPSLA 2016). ACM, New York, NY, USA, 74-91. https://doi.org/10. $1145 / 2983990.2984027$

[63] Ken Thompson. 1984. Reflections on Trusting Trust. Commun. ACM 27, 8 (Aug. 1984), 761-763. https://doi.org/10.1145/358198.358210

[64] Nickolai Zeldovich, Silas Boyd-Wickizer, Eddie Kohler, and David Mazières. 2006. Making Information Flow Explicit in HiStar. In Proceedings of the 7th USENIX Symposium on Operating Systems Design and Implementation - Volume 7 (OSDI '06). USENIX Association, Berkeley, CA, USA, 19-19. http://dl.acm.org/citation.cfm?id=1267308.1267327 